\title{
Background risk and the demand for state-contingent claims ${ }^{\star}$
}

\author{
Guenter Franke $^{1}$, Richard C. Stapleton ${ }^{2}$, and Marti G. Subrahmanyam ${ }^{3}$ \\ 1 Fakultät für Wirtschaftswissenschaften und Statistik, University of Konstanz, 78457 Konstanz, \\ GERMANY (e-mail: guenter.franke@uni-konstanz.de) \\ 2 University of Strathclyde, Strathclyde, UNITED KINGDOM and \\ University of Melbourne, Melbourne, AUSTRALIA \\ (e-mail: richard.stapleton1@btinternet.com) \\ 3 Stern School of Business, New York University, New York, USA \\ (e-mail: msubrahm@stern.nyu.edu)
}

Received: February 13, 2002; revised version: February 10, 2003

Summary. We consider the demand for state-contingent claims, in the presence of an independent zero-mean, non-hedgeable background risk. An agent is defined to be generalized risk averse if he/she chooses a demand function for contingent claims with a smaller slope everywhere, given a simple increase in background risk. We show that the conditions for standard risk aversion, that is positive, declining absolute risk aversion and prudence, are necessary and sufficient for generalized risk aversion.

Keywords and Phrases: Background risk, Precautionary premium, Demand for tradable risk.

JEL Classification Numbers: D52, D81, G11.

\section{Introduction}

Economic agents often face both tradable and non-tradable risks. Examples of tradable risks are the risks in foreign exchange and interest-rate markets. Examples of risks that may be non-tradable are the risk of labor income and the risk of production losses. These non-tradable risks are called background risks.

As an example, consider the effect of labor-income risk on the portfolio choice of an investor. There are three levels at which this risk can have an impact. First, to

\footnotetext{
* We are grateful to Louis Eeckhoudt, Christian Gollier, Harris Schlesinger and an unknown referee for valuable comments.

Correspondence to: G. Franke
} 
the extent that labor income has a positive mean, this affects the endowment of the investor. For example, a positive non-stochastic labor income increases the endowment of the investor and thereby affects his/her risk aversion. Second, to the extent that a stochastic labor income is correlated with the return on investments available in the market, the background risk of labor income creates a hedging demand for the marketable investments. The investor will buy stocks which are negatively correlated with his/her labor income. At a third level, the pure (orthogonal) component of the labor-income risk can induce precautionary behaviour of the investor. It is this third effect that has been analysed in the background risk literature, by studying the impact of an independent, zero-mean risk on the demand of an agent for marketable risks. The fact that this third type of effect can have a significant influence in a broad range of problems has been highlighted, for example, by Franke, Stapleton and Subrahmanyam (1998) in the case of portfolio demand and Weil (1992) in the case of asset prices. Various papers have analysed the impact of certain types of increases in background risk on the demand for insurance, where the amount of insurance is measured by the coinsurance rate and the deductible (see, for example Eeckhoudt and Kimball, 1992; Meyer and Meyer, 1998).

Recent advances in the theory of risk bearing have concentrated on the effect of a non-tradable background risk on the risk aversion of an agent to a second independent risk. For example, Gollier and Pratt (1996) define a rather general class of utility functions such that risk-averse individuals become even more risk averse towards a risk, when a second, independent, unfair background risk is added. They compare the risk aversion of an agent with no background risk to that of an agent who faces the background risk. They term the set of functions under which the agent becomes more risk averse, the class of "risk-vulnerable" utility functions. The set of risk-vulnerable functions is larger than the set of proper risk averse functions introduced earlier by Pratt and Zeckhauser (1987), who consider utility functions such that the expected utility of an undesirable risk is decreased by the presence of an independent, undesirable risk. Kimball (1993) has considered the effect of the [even larger] set of expected marginal utility increasing background risks. This led him to define the more restrictive class of standard risk averse utility functions. Standard risk aversion characterizes those functions where the individual responds to an expected marginal utility increasing background risk by reducing the demand for a marketed risk. Kimball shows that standard risk averse functions are characterized by positive, decreasing absolute risk aversion and absolute prudence. The set of standard risk averse functions is a subset of the set of proper risk averse functions, which, in turn, are a subset of the risk vulnerable functions, as discussed by Gollier and Pratt (1996, pp. 1118-1119). In a related paper, Eeckhoudt, Gollier and Schlesinger (1996) extend this analysis by considering a rather general set of changes in background risk, which take the form of first or second order stochastic dominance changes. They establish a set of quite restrictive conditions on the utility function such that agents become more risk averse when background risk increases in this sense.

The existing literature discusses the conditions under which an agent reacts to background risk by buying less of a risky asset, when faced with the choice between a single risky asset and a risk-free asset. Such a risky asset can be thought of as 
a portfolio of state-contingent claims. We consider an economy where the agent can buy state-contingent claims individually. We then ask the question: how is the agent's demand for these claims affected by an increase in background risk? In a state-contingent claims model, risk averse behaviour can be characterized by the slope of the demand curve for state-contingent claims. For example, in the limiting case of extreme risk aversion, the agent buys an equal amount of claims on each state, regardless of the price of the claims. A less risk-averse agent buys a schedule of claims weighted towards claims that are relatively cheap, i.e., a downward sloping demand curve. Intuitively, it seems reasonable, in this economy, to characterize more risk-averse behaviour as having a flatter demand curve for state-contingent claims.

The purpose of this paper is to investigate restrictions on utility functions which guarantee more risk averse behaviour in the presence of an increased, independent, zero-mean background risk, when the agent faces a choice between state-contingent claims. Agents are said to be generalized risk averse, if they react to a simple increase in background risk, by changing their demand for contingent claims in such a way that the slope of the demand function becomes smaller everywhere. A simple increase in risk is a deterministic, monotonic transformation in risk as defined by Meyer and Ormiston (1989). In this paper we analyse the set of simple increases in background risk. We use the concept of generalized risk aversion to extend risk vulnerability in two ways. First, instead of comparing a positive and a zero level of background risk, simple increases in risk are analysed. Second, instead of studying the impact of an increase in background risk on derived risk aversion, we examine the impact of an increase in background risk on the demand for state-contingent claims.

The research question is: what is the set of utility functions inducing generalized risk aversion? Equivalently, what are the necessary and sufficient properties of utility functions for a simple increase in background risk to reduce the slope of the demand curve for state-contingent claims everywhere? In Section 2, we define the concepts of generalized risk aversion and simple increases in background risk which are central to the analysis of the paper. In Section 3, we present our main result. Agents are generalized risk averse if and only if they have positive declining absolute risk aversion and prudence. These conditions are precisely the conditions for standard risk aversion. They are sufficient for the slope of the demand function for state-contingent claims to become smaller everywhere. What is more surprising is that these conditions are also necessary for generalized risk aversion. Necessity arises from the fact that the slope of the demand function for contingent claims must become less steep at all levels. As Kimball argues, declining absolute risk aversion and declining absolute prudence are natural attributes of the utility function. They are shared, also, by the HARA class of functions with an exponent less than one. The larger set of risk-vulnerable utility functions, used by Gollier and Pratt, is not restrictive enough, when we consider the effect of simple increases in background risk on the slope of the demand function. Our result therefore adds to the case for the standard risk-averse functions to be the natural class of functions to use when analysing the impact of background risk. 


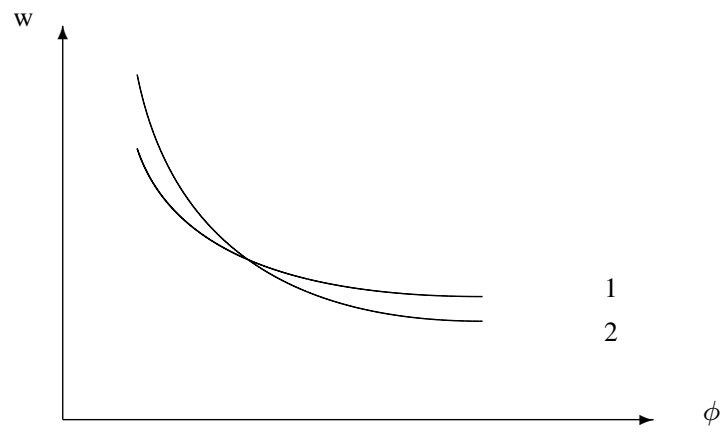

Figure 1. This figure shows the demand curves for state-contingent claims for two different levels of background risk. Demand curve 1 represents a higher level of background risk than 2, and is, therefore, less steep

\section{Generalized risk aversion}

Gollier (2001) considers a model where the agent can buy state-contingent claims on consumption, given no background risk. Let $\phi$ be the probability deflated price of obtaining one unit of consumption if a state occurs and obtaining nothing otherwise. Then, in this model, the higher is $\phi$ for a given state, the lower is the agent's demand for claims on that state, $w$. In other words, the demand function, $w(\phi)$, that relates the consumption in a state to the price, is downward sloping. Gollier [Proposition 51] shows that, if two agents with utility functions $u_{1}$ and $u_{2}$ have the same endowment, and if $u_{1}$ is more risk averse than $u_{2}$, then the demand function of agent $1, w_{1}(\phi)$, 'single-crosses from below' the demand function of agent 2 , $w_{2}(\phi)$. This single-crossover property is illustrated in Figure 1. Gollier goes on to conclude that "risk-vulnerable investors will select a safer consumption plan", when they face background risk. Hence, if a simple increase in background risk raises risk aversion, then an agent facing an increase in background risk will respond by choosing a demand function similar to investor 1 rather than that chosen by investor 2, in Figure 1.

However, Gollier's analysis highlights a problem. Even though agent 1 is more risk averse than agent 2 , he could have a demand function that has a smaller slope at the crossover point, but has a greater slope over some range of $\phi$. This means that the more risk-averse investor actually exhibits less risk-averse behaviour over some range . As Gollier notes, the single-crossover property only throws light on local risk-taking behaviour in the range around the crossover point. In this paper, we wish to look at local risk-taking behaviour over all ranges, hence we employ a stricter definition of more risk-averse behaviour in the state-contingent claims model.

If the agent responds to a simple increase in background risk by choosing a demand function with a smaller slope everywhere, we say that the agent is generalized risk averse. This concept of generalized risk aversion relates closely to the previously discussed concepts of 'risk vulnerability' and 'standard risk aversion'. 
In the case of 'risk vulnerability', an agent responds to the introduction of background risk by reducing his demand for a single risky asset. In the case of standard risk aversion, an agent responds to a marginal utility-increasing background risk in the same manner. In the case of generalized risk aversion, the idea of the response of risk-taking behaviour to a simple increase in background risk is extended to the demand for state-contingent claims.

We consider the effect of an independent background risk on the demand for state-contingent claims, using an extension of the analysis of Back and Dybvig (1993), who establish conditions for the optimality of an agent's demand. We investigate the set of [restrictions on] utility functions such that the agent responds to simple increases in a zero-mean background risk by choosing a demand function that has a smaller slope at all price levels. In the context of this choice problem, we need to restrict the set of changes in background risk to the set of simple increases as defined by Meyer and Ormiston (1989). We follow this terminology here. ${ }^{1}$

Definition 1 (Simple Increases in Background Risk) Let y be a background risk with $E(y)=0$. Then a simple increase in the background risk changes $y$ to $y+s \Delta(y)$, with $E[\Delta(y)]=0$, where $\Delta(y)$ is non-decreasing and continuous in $y$, and where $s \geq 0$ represents the scale of the increase.

Note that simple increases in background risk leave the rank order of outcomes unchanged. Continuity of the transformation function $\Delta(y)$, together with the condition $E[\Delta(y)]=0$, guarantees that there exists a $y_{0}$ such that $\Delta\left(y_{0}\right)=0$. Instead of continuity, we could assume directly the existence of $y_{0}$. In the following analysis, we differentiate with respect to $s$ to derive the effects of a marginal increase in background risk.

A simple increase in background risk replaces $y$ by $y+s \Delta(y)$, where $\partial \Delta / \partial y \geq$ $0, \forall y$, and where $E[\Delta(y)]=0$. Hence, a simple increase in background risk is a change, $\Delta(y)$, that itself increases with $y$. An example of a simple increase is a proportionate increase where $\Delta(y)$ is proportionate to $y$. We can now formally define generalized risk aversion:

Definition 2 An agent is generalized risk-averse if the absolute value of the slope of his/her demand function for state-contingent claims $w(\phi)$ becomes smaller for all $\phi$, given a simple increase in background risk.

We consider an agent who can buy a set of contingent claims on future consumption and faces background risk. The agent's total income at the end of the period, $W$, is therefore composed of an income from tradeable claims, $w$, plus the background risk $y$, i.e. $W=w+y$. We assume that background risk, $y$, has a zero mean, and is bounded from below, $y \geq a$. We also assume that total income, $W$, has a lower bound, $\underline{\mathrm{W}}$. Hence, $w>\underline{W}-a$. Moreover we assume that $y$ is distributed independently of $w$. A state of the world determines both the agent's income from tradeable claims and the background risk. Let $(\Omega, \mathcal{F}, \mathcal{P})$ be the probability space on which the random variables are defined.

\footnotetext{
1 Note that this is in contrast to the use by Eeckhoudt, Gollier and Schlesinger (1995) of the term 'a
} simple spread across $\alpha$, which allows for more general increases in risk. 
The agent's utility function is $u(W)$. We assume that the utility function is stateindependent, strictly increasing, strictly concave, and four times differentiable on $W \varepsilon(\underline{W}, \infty)$. We assume that there exist integrable functions on $\omega \varepsilon \Omega, u_{0}$ and $u_{1}$ such that

$$
u_{0}(\omega) \leq u(W) \leq u_{1}(\omega)
$$

We also assume that similar conditions hold for the derivatives $u^{\prime}(W), u^{\prime \prime}(W)$ and $u^{\prime \prime \prime}(W)$. The agent's expected utility, conditional on $w$, is given by the derived utility function, as defined by Kihlstrom et al. (1981) and Nachman (1982):

$$
\nu(w)=E_{y}[u(W)] \equiv E[u(w+y) \mid w]
$$

where $E_{y}$ indicates an expectation taken over different outcomes of $y$. Thus, the agent with background risk and a von Neumann-Morgenstern concave utility function $u(W)$ acts like an individual without background risk and a concave utility function $\nu(w){ }^{2}$ The coefficient of absolute risk aversion is defined as $r(W)=-u^{\prime \prime}(W) / u^{\prime}(W)$ and the coefficient of absolute prudence as $p(W)=$ $-u^{\prime \prime \prime}(W) / u^{\prime \prime}(W)$. From Kimball (1993), the agent is standard risk averse if and only if $r(W)$ and $p(W)$ are both positive and declining. The absolute risk aversion of the agent's derived utility function is defined as the negative of the ratio of the second derivative to the first derivative of the derived utility function with respect to $w$, i.e.,

$$
\hat{r}(w)=-\frac{\nu^{\prime \prime}(w)}{\nu^{\prime}(w)}=-\frac{E_{y}\left[u^{\prime \prime}(W)\right]}{E_{y}\left[u^{\prime}(W)\right]}
$$

It is worth noting that, in the absence of background risk, $\hat{r}(w)$ is equal to $r(W)$, the coefficient of absolute risk aversion of the original utility function.

\section{The effect of changes in background risk on the optimal demand function for state-contingent claims}

In this section we derive the necessary and sufficient condition for the utility function to exhibit generalized risk aversion. We assume that the capital market is perfect. A state of nature determines both the agent's tradable income $w$ and his background risk income $y$. We partition the state space into subsets of states that differ only in the background risk, $y$. We call these subsets "traded states" since they represent states on which state-contingent claims can be traded. We assume there is a continuum of such states and, for convenience, we label these states by a continuous variable $x \in R^{+}$. We assume the market, in the traded states, is complete. We also assume that there exists a pricing kernel, $\phi=\phi(x)$ with the property $\phi>0$, where $\phi(x)$ is a continuous function. ${ }^{3}$

\footnotetext{
2 See, for example, Eeckhoudt, Gollier and Schlesinger (1996), p. 684.

3 The market is complete in the sense of Nachman (1988). The agent can buy a digital option which pays one unit of consumption, if $x \geq k$, and 0 otherwise, $\forall k \in R^{+}$. The price of such an option is
}

$$
\int_{k}^{\infty} \phi(x) f(x) d(x)
$$


Let $w=g(x)$ be the agent's income from the purchase of state-contingent claims. The agent chooses $w=g(x)$, subject to the constraint that the cost of acquiring this set of claims is equal to his/her initial endowment. The background risk $y$ affects his/her choice of the function $w=g(x)$. We assume that the agent has sufficient endowment to ensure that $w$ can be chosen to obtain $W>\underline{W}$ in all states. We also assume certain properties of the utility function, in addition to those assumed in the previous section. First, the marginal utility has the limits:

$$
\begin{gathered}
u^{\prime}(W) \rightarrow \infty \text { if } W \rightarrow \underline{W}, \\
u^{\prime}(W) \rightarrow 0 \text { if } W \rightarrow \infty .
\end{gathered}
$$

Second, the risk aversion goes to zero at high levels of income, i.e.

$$
r(W) \rightarrow 0 \text { if } W \rightarrow \infty .
$$

These reasonable restrictions are satisfied, for example, by the HARA class with an exponent less than 1.

The agent solves the following maximization problem $^{4}$ :

$$
\begin{gathered}
\quad \max _{w=g(x)} E_{x}[\nu(w)]=E_{x}[\nu(g(x))] \\
\text { s.t. } \quad E_{x}\left[\left(g(x)-g^{0}(x)\right) \phi(x)\right]=0
\end{gathered}
$$

In the budget constraint, $w^{0}=g^{0}(x)$ is the agent's endowment of claims. $\phi(x)$, the pricing kernel, is given exogenously, with $E[\phi(x)]=1$. The maximisation problem (3) is a standard state-preference maximisation problem. The expectation, $E_{x}($.$) , is taken only over the traded states. Note that the background risk has only an$ indirect impact on problem (3) through its effect on the derived utility function. This is defined by equation (1) as the expected value of utility over different outcomes of $y$, given the traded income $w$.

The first order condition for a maximum is

$$
\nu^{\prime}(g(x))=\lambda \phi(x),
$$

or simply

$$
\nu^{\prime}(w)=\lambda \phi,
$$

where $\lambda$ is a positive Lagrange multiplier which reflects the tightness of the budget constraint. Equation (4) holds as an equality since, by assumption, $u^{\prime}(W) \rightarrow \infty$ for $W \rightarrow \underline{W}$ and $u^{\prime}(W) \rightarrow 0$ for $W \rightarrow \infty$. The demand for claims in equation (4) can be shown to be optimal and unique under some further finiteness restrictions. ${ }^{5}$ This follows from the results of Back and Dybvig (1993).

where $\phi(x)$ is the pricing kernel and the probability density function is $f(x)$. A contingent claim is a contract (a portfolio of digital options) paying one unit of consumption if $x \epsilon[k, k+\eta)$ and nothing otherwise, for positive, infinitely small $\eta$.

${ }^{4}$ See also Hirshleifer and Riley (1992, p.46) for a discussion of the demand for state-contingent claims.

${ }^{5} E[w \phi]<\infty$ for any $\lambda>0$ and each $w$ satisfying (4) is assumed. 
From the first order condition (4), it follows that we can define a function $w=w(\phi)=\nu^{\prime(-1)}(\lambda \phi)$. Hence, given the derived utility function and the initial endowment, the demand for claims contingent on a traded state $x$ depends only on $\phi(x)$. Thus $w(\phi)$ is a deterministic function relating the demand for state-contingent claims to the pricing kernel. It follows from our assumptions that $\partial w / \partial \phi$ and $\partial^{2} w / \partial \phi \partial s$ exist. $^{6}$

Our aim is to find the necessary and sufficient conditions on the utility function, which guarantee that the agent's demand function becomes less steep given a simple increase in background risk, i.e. the agent is generalized risk averse. It will be shown that the conditions for generalized risk aversion are the same as those for standard risk aversion.

Differentiating equation (4) with respect to $\phi$, for a given level of background risk, and dividing by $\lambda \phi$, yields the slope of the demand function

$$
\frac{\partial w}{\partial \phi}=\frac{-1 / \phi}{\hat{r}(w)}, \forall(\phi, y)
$$

Suppose that background risk increases the derived risk aversion of the agent, $\hat{r}(w)$. It follows from equation (5) that the background risk affects the slope of the demand function. We now consider the effect of changes in the level of background risk, assuming that the pricing function $\phi(x)$ is given. From equation (5) it appears at first sight that the slope of the demand function becomes less steep whenever the increase in background risk increases the agent's derived risk aversion. In fact, it follows from Gollier (2001, Proposition 51) that:

Proposition 1 Suppose that an increase in background risk raises the agent's derived risk aversion everywhere. Then the new demand curve for state-contingent claims intersects the original one once from below. ${ }^{7}$

Proof. At an intersection of the new demand curve, $w_{1}(\phi)$, and the original demand curve, $w_{2}(\phi), w_{1}=w_{2}$ so that, by equation (5), $\partial w_{1} / \partial \phi>\partial w_{2} / \partial \phi$ follows from $\hat{r}_{1}>\hat{r}_{2}$. A second intersection would require $\partial w_{1} / \partial \phi<\partial w_{2} / \partial \phi$, which contradicts (5). Also, at least one intersection must exist, in order for the budget constraint to be satisfied.

However, as noted by Gollier (2001), the one-intersection property does not imply that the new demand curve is less steep than the original one everywhere.

6 Consider the function

$$
F(w, \phi)=\nu^{\prime}(w)-\lambda \phi=0 .
$$

The partial derivative $F_{w}$ exists and is continuous, since the utility function $u(w+y)$ and its first three derivatives are assumed to exist and to be integrable. Also $F_{w} \neq 0$ for $w<\infty$. Hence, by the implicit function theorem, the function $w=w(\phi)$ is differentiable with

$$
\partial w / \partial \phi=-\frac{F_{\phi}}{F_{w}} .
$$

Also, since $y$ is differentiable in $s$, and since $F_{\phi}$ and $F_{w}$ are differentiable in $y$, then $F_{\phi}$ and $F_{w}$ are also differentiable in $s$. It follows that $\partial^{2} w / \partial \phi \partial s$ also exists.

7 The one intersection property is illustrated in Figure 1. 
This is because a change in background risk, affects $\hat{r}(w)$ both directly and through the induced change in $w .^{8}$ The main result of the paper is Proposition (2).

Proposition 2 (Generalized risk aversion) Assume any simple increase in an independent, zero-mean background risk. Let $u^{\prime}(W)>0$ and $u^{\prime \prime}(W)<0$, where $W \varepsilon(\underline{W}, \infty)$. Suppose that $u^{\prime}(W) \rightarrow \infty$ for $W \rightarrow \underline{W}$ and that $u^{\prime}(W) \rightarrow 0$ and $r(W) \rightarrow 0$, for $W \rightarrow \infty$. Then

$$
\frac{d}{d s}\left[\frac{\partial w}{\partial \phi}\right] \geq 0, \forall(\phi, y, s)
$$

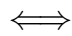

the utility function is standard risk averse.

We first establish three lemmas which are required in the proof. We have

Lemma 1 Suppose that $u^{\prime}(W) \rightarrow \infty$ for $W \rightarrow \underline{W}$, then $r(W) \rightarrow \infty$ and $p(W) \rightarrow \infty$ for $W \rightarrow \underline{W}$.

Proof. $u^{\prime}(W) \rightarrow \infty$, for $W \rightarrow \underline{W}$, implies $\partial \ln u^{\prime}(W) / \partial W \rightarrow-\infty^{9}$ and hence $r(W) \rightarrow \infty$. Also, since for $W \rightarrow \underline{W}, r^{\prime}<0, p>r$, and hence $p(W) \rightarrow \infty$.

The second lemma establishes the equivalence of declining risk aversion and declining derived risk aversion. We have:

Lemma $2 \hat{r}^{\prime}(w) \leq 0$ for any background risk $\Leftrightarrow r^{\prime}(W) \leq 0$.

Proof. Kihlstrom et al. (1981) and Nachman (1982) have shown that declining risk aversion implies declining derived risk aversion. Conversely, declining derived risk aversion implies declining risk aversion of $u(W)$. This follows from the case of small background risks.

The third lemma establishes a condition for declining prudence, in the case of simple changes in background risk:

Lemma 3 For simple increases in background risk,

$$
\frac{d}{d \phi}\left[-\frac{\partial \nu^{\prime}(w) / \partial s}{\partial \nu^{\prime}(w) / \partial w}\right] \geq 0 \Leftrightarrow p^{\prime}(W) \leq 0
$$

Proof. See Appendix.

8 Differentiating equation (5) once more with respect to $\phi$ reveals that declining absolute risk aversion implies convexity of $w(\phi)$. In addition, as pointed out by the referee, to the left of the crossover point in Figure $1, w_{1}<w_{2}$ implies that $\hat{r}_{1}\left(w_{1}\right) \geq \hat{r}_{2}\left(w_{2}\right)$, and hence $\partial w_{1} / \partial \phi \geq \partial w_{2} / \partial \phi$ by equation (5). However, to the right of the crossover point, declining risk aversion is not sufficient to guarantee the result.

9 Starting from a finite value of $u^{\prime}(w), u^{\prime}(w) \rightarrow \infty$ for $w \rightarrow \underline{W}$ requires that the growth rate of $u^{\prime}(w) \rightarrow-\infty$. 
We now present the proof of Proposition (2).

Proof of Proposition (2). Totally differentiating equation (4) with respect to $s$ yields

$$
\frac{\partial \nu^{\prime}(w)}{\partial s}+\frac{\partial \nu^{\prime}(w)}{\partial w} \frac{\partial w}{\partial s}=\frac{d \lambda}{d s} \phi
$$

Substituting $\lambda$ from equation (4) then yields

$$
\frac{\partial \nu^{\prime}(w)}{\partial s}+\frac{\partial \nu^{\prime}(w)}{\partial w} \frac{\partial w}{\partial s}=\frac{d \ln \lambda}{d s} \nu^{\prime}(w) .
$$

Hence, the effect of the background risk on the demand for claims is given by

$$
\frac{\partial w}{\partial s}=-\frac{d \ln \lambda}{d s} \frac{1}{\hat{r}(w)}-\frac{\partial \nu^{\prime}(w) / \partial s}{\partial \nu^{\prime}(w) / \partial w} .
$$

The Proposition is concerned with the conditions under which

$$
\frac{d}{d s}\left[\frac{\partial w}{\partial \phi}\right]=\frac{d}{d \phi}\left[\frac{\partial w}{\partial s}\right] \geq 0 .
$$

We investigate these conditions by looking at the behaviour of the two terms in equation (7).

Sufficiency of standard risk aversion. First, we show that the first term in (7) is negative, while the second term is positive. In order to satisfy the budget constraint, $\partial w / \partial s$ has to be positive in some traded states and negative in others. Given positive prudence, $\partial \nu^{\prime}(w) / \partial s>0$, so that the second term in (7) is positive. It follows that the first term must be negative, i.e. $\frac{d \ln \lambda}{d s} \geq 0$, hence $\frac{d \lambda}{d s} \geq 0$. We can now investigate

$$
\frac{d}{d \phi}\left[\frac{\partial w}{\partial s}\right]
$$

by taking the two terms in (7) one-by-one. First, the (negative) first term increases with $\phi$, since

$$
\frac{\partial \hat{r}}{\partial \phi}=\frac{\partial \hat{r}}{\partial w} \frac{\partial w}{\partial \phi}
$$

is positive. This follows from $\partial w / \partial \phi<0$ (see equation (5)) and $\partial \hat{r} / \partial w \leq 0$ (which in turn follows from $\partial r / \partial w \leq 0$ and Lemma 2). Second, the (positive) second term increases in $\phi$, given declining prudence (see Lemma 3 ). Hence

$$
\frac{d}{d \phi}\left[\frac{\partial w}{\partial s}\right]
$$

is positive given standard risk aversion. 
Necessity of standard risk aversion. We establish necessity of standard risk aversion by taking the special case of a small background risk. Also, we assume $\phi$ converges in probability to a degenerate distribution, $\phi_{0}$. By assuming $w\left(\phi_{0}\right)$ is, in turn, large [small], we show that the first [second] term in (7) dominates. For the first term in (7) to increase in $\phi$, declining risk aversion is required. For the second term in (7) to increase in $\phi$, declining prudence is required. Hence, to cover both of these possibilities, standard risk aversion is required. First, we consider the term $-d \ln \lambda / d s$.

We have from equation (4),

$$
E\left[\nu^{\prime}(w)\right]=E\left[u^{\prime}(w+y)\right]=E(\lambda \phi)=\lambda
$$

and

$$
\frac{d \lambda}{d s}=\frac{d}{d s} E\left[u^{\prime}(w+y)\right]=\frac{d}{d s} E\left[u^{\prime}(w-\psi)\right],
$$

where $\psi=\psi(w)$ is the precautionary premium as defined by Kimball(1990). Hence,

$$
\frac{d \lambda}{d s}=E\left\{u^{\prime \prime}(w-\psi)\left[\frac{\partial w}{\partial s}-\frac{\partial \psi}{\partial s}-\frac{\partial \psi}{\partial w} \frac{\partial w}{\partial s}\right]\right\} .
$$

Assume that we start from a position of no background risk, $s=0$. In this case, $\psi=0$, and $\partial \psi / \partial w=0$. Since, for small background risks with variance $\sigma^{2}$, the precautionary premium is ${ }^{10}$

$$
\psi=\frac{1}{2} p(w) \sigma^{2}
$$

we have

$$
\frac{\partial \psi}{\partial s}=\frac{1}{2} p(w) \frac{\partial \sigma^{2}}{\partial s}
$$

It follows that

$$
\frac{d \lambda}{d s}=E\left\{u^{\prime \prime}(w)\left[\frac{\partial w}{\partial s}-\frac{\partial \psi}{\partial s}\right]\right\}=E\left\{u^{\prime \prime}(w)\left[\frac{\partial w}{\partial s}-\frac{1}{2} p(w) \frac{\partial \sigma^{2}}{\partial s}\right]\right\} .
$$

Now we assume that $\phi$ converges to the degenerate distribution $\phi_{0}$, in probability. Since we can write

$$
\frac{d \lambda}{d s}=E[f(\phi)]
$$

where $f$ is a continuous, uniformly integrable function, then it follows that

$$
\frac{d \lambda}{d s} \rightarrow u^{\prime \prime}\left(w_{0}\right)\left[-\frac{1}{2} p\left(w_{0}\right) \frac{\partial \sigma^{2}}{\partial s}\right]
$$

where $w_{0}=w\left(\phi_{0}\right)$, since $\partial w_{0} / \partial s=0$, for the case of the degenerate distribution, $\phi_{0}$. Dividing by $\lambda=u^{\prime}\left(w_{0}\right)$,

$$
\frac{d \ln \lambda}{d s} \rightarrow \frac{u^{\prime \prime}\left(w_{0}\right)}{u^{\prime}\left(w_{0}\right)}\left[-\frac{1}{2} p\left(w_{0}\right) \frac{\partial \sigma^{2}}{\partial s}\right]
$$

10 This follows by analogy with the Pratt-Arrow argument for the risk premium, since initially there is no background risk. 
and hence

$$
-\frac{d \ln \lambda}{d s} \rightarrow-r\left(w_{0}\right)\left[\frac{1}{2} p\left(w_{0}\right) \frac{\partial \sigma^{2}}{\partial s}\right] .
$$

Substituting in (7), we now have

$$
\frac{\partial w}{\partial s} \rightarrow r\left(w_{0}\right)\left[\frac{1}{2} p\left(w_{0}\right) \frac{\partial \sigma^{2}}{\partial s}\right] \frac{-1}{\hat{r}(w)}-\frac{\partial \nu^{\prime}(w) / \partial s}{\partial \nu^{\prime}(w) / \partial w} .
$$

Starting with no background risk, the term

$$
-\frac{\partial \nu^{\prime}(\cdot) / \partial s}{\partial \nu^{\prime}(\cdot) / \partial w}=\frac{1}{2} p(w) \frac{\partial \sigma^{2}}{\partial s}
$$

since $\partial \psi / \partial w=0$. Hence, we can write

$$
\frac{\partial w}{\partial s} \rightarrow r\left(w_{0}\right)\left[\frac{1}{2} p\left(w_{0}\right) \frac{\partial \sigma^{2}}{\partial s}\right] \frac{-1}{\hat{r}(w)}+\frac{1}{2} p(w) \frac{\partial \sigma^{2}}{\partial s} .
$$

Differentiating (8) with respect to $\phi$, we then have

$$
\frac{d}{d s}\left[\frac{\partial w}{\partial \phi}\right]=\frac{d}{d \phi}\left[\frac{\partial w}{\partial s}\right] \rightarrow\left\{r\left(w_{0}\right)\left[\frac{1}{2} p\left(w_{0}\right) \frac{\partial \sigma^{2}}{\partial s}\right] \frac{\hat{r}^{\prime}(w)}{\hat{r}(w)^{2}}+\frac{1}{2} p^{\prime}(w) \frac{\partial \sigma^{2}}{\partial s}\right\} \frac{\partial w}{\partial \phi}
$$

Since $\partial w / \partial \phi<0$, the condition for a smaller slope becomes

$$
r\left(w_{0}\right)\left[\frac{1}{2} p\left(w_{0}\right) \frac{\partial \sigma^{2}}{\partial s}\right] \frac{\hat{r}^{\prime}(w)}{\hat{r}(w)^{2}}+\frac{1}{2} p^{\prime}(w) \frac{\partial \sigma^{2}}{\partial s} \leq 0 .
$$

To establish the necessity of declining absolute risk aversion, we choose $\phi_{0}$ such that $w_{0} \rightarrow \underline{W}$. By Lemma 1, hence, $r(w) \rightarrow \infty$ and $p\left(w_{0}\right) \rightarrow \infty$, for $w \rightarrow \underline{W}$. Therefore, $\hat{r}^{\prime}(w)>0$ implies that the first term in equation (9) $\rightarrow \infty$. Then, since the second term in (9) is independent of $w_{0}, \hat{r}^{\prime} \leq 0$ and by Lemma $2, r^{\prime} \leq 0$ is required for the condition (9) to hold. $r^{\prime} \leq 0$ also establishes the necessity of positive prudence, $p>0$.

To establish necessity of declining absolute prudence, we choose $\phi_{0}$ such that $w_{0} \rightarrow \infty$ and hence, by assumption, $r\left(w_{0}\right) \rightarrow 0$. Then $r^{\prime}\left(w_{0}\right)=r\left(w_{0}\right)\left[r\left(w_{0}\right)-\right.$ $\left.p\left(w_{0}\right)\right] \rightarrow 0$ implies $r\left(w_{0}\right) p\left(w_{0}\right) \rightarrow 0$. Hence the first term in equation (9) $\rightarrow 0$. Then, since the second term in (9) is independent of $w_{0}, p^{\prime} \leq 0$ is required for the condition (9) to hold. Hence standard risk aversion is a necessary condition for a smaller slope.

Proposition 2 allows us to analyze the effect of any marginal simple increase in a zero-mean, independent background risk, given that this increase has a negligible impact on the prices of state-contingent claims. Since a finite increase in background risk is the sum of marginal increases, the sufficiency condition in Proposition 2 also holds for finite increases in background risk. Proposition 2 says that a simple increase in background risk will reduce the steepness of the slope of this agent's demand function everywhere. As can be seen from Figure 1, the agent reacts to a simple increase in background risk by purchasing more claims in traded states for which the price $\phi$ is high, financing the purchase by selling some claims in the traded 
states with low prices. Proposition 2 can also be interpreted by comparing, within an equilibrium, the demand of agents, who differ only in the size of their respective background risks. Proposition 2 suggests that agents with higher background risk will adjust their demand functions by buying state-contingent claims on high-price traded states and selling claims on low-price traded states. This is illustrated in Franke, Stapleton and Subrahmanyam (1998), for an economy in which all agents have the same type HARA-class utility function, exhibiting declining absolute risk aversion. These functions are standard risk averse and hence generalized risk averse. In this economy, agents with high background risk buy options from those with relatively low background risk. The latter agents sell portfolio insurance to the former with relatively high background risk.

\section{Conclusions}

The main conclusions regarding the effects of a simple increase in background risk, on the demand for state-contingent claims and on derived risk aversion, are summarised in Propositions 1 and 2 of the paper. Proposition 2 provides the necessary and sufficient conditions for simple increases in background risk to reduce the slope of an agent's demand function for state-contingent claims everywhere (generalized risk aversion). These conditions are the same as those for standard risk aversion. Essentially, to guarantee a lower slope at high [low] prices of state-contingent claims, positive and declining absolute risk aversion [prudence] is required.

It is interesting to compare this result with that of Kimball (1993). Kimball also considers a comparative statics question: when does a marginal utility increasing risk raise derived risk aversion? This set of risks includes those that are non-stochastic and have a negative mean as well as those of high risk and zero or positive mean. Hence both positive and declining risk aversion and prudence are required. We restrict ourselves to simple increases in background risk, but ask the more demanding question about the effect on the demand curve for state-contingent claims. It turns out that the conditions for generalized risk aversion are the same as for standard risk aversion.

\section{Appendix}

\section{Proof of Lemma (3)}

We have to show that

$$
-\frac{\partial \nu^{\prime}(w) / \partial s}{\partial \nu^{\prime}(w) / \partial w}
$$

increases in $\phi$, if and only if absolute prudence is declining. This term increases in $\phi$ if the negative of the term increases in $w$, since $\partial w / \partial \phi<0$. In terms of the underlying utility function, this is the same as showing that the term

$$
Z(w)=\frac{E_{y}\left[u^{\prime \prime}(W) \Delta(y)\right]}{E_{y}\left[u^{\prime \prime}(W)\right]}
$$

is increasing in $w$. 
Now consider a marginal increase in $w$. Then

$\operatorname{sgn} \frac{\partial Z(w)}{\partial w}=\operatorname{sgn} E_{y}\left[u^{\prime \prime}(W)\right] E_{y}\left[u^{\prime \prime \prime}(W) \Delta(y)\right]-E_{y}\left[u^{\prime \prime \prime}(W)\right] E_{y}\left[u^{\prime \prime}(W) \Delta(y)\right]$,

or,

$$
\operatorname{sgn} \frac{\partial Z(w)}{\partial w}=\operatorname{sgn}-E_{y}\left[\left\{u^{\prime \prime \prime}(W)-u^{\prime \prime}(W) \frac{E_{y}\left[u^{\prime \prime \prime}(W)\right]}{E_{y}\left[u^{\prime \prime}(W)\right]}\right\} \Delta(y)\right]
$$

and then it follows that

$$
\operatorname{sgn} \frac{\partial Z(w)}{\partial w}=\operatorname{sgn}-E_{y}\left[\left\{u^{\prime \prime \prime}(W)-u^{\prime \prime}(W) \frac{E_{y}\left[u^{\prime \prime \prime}(W)\right]}{E_{y}\left[u^{\prime \prime}(W)\right]}\right\}(\Delta(y)-\Delta(\hat{y}))\right]
$$

where $\hat{y}$ is defined by

$$
\frac{E_{y}\left[u^{\prime \prime \prime}(W)\right]}{E_{y}\left[-u^{\prime \prime}(W)\right]}-p(\hat{W}=w+\hat{y})=0
$$

Hence

$$
\operatorname{sgn} \frac{\partial Z(w)}{\partial w}=\operatorname{sgn} E_{y}\left[-u^{\prime \prime}(W)\left\{\frac{E_{y}\left[u^{\prime \prime \prime}(W)\right]}{E_{y}\left[-u^{\prime \prime}(W)\right]}-p(W)\right\}(\Delta(y)-\Delta(\hat{y}))\right]
$$

Simple increases in background risk imply that

$$
\Delta(y)-\Delta(\hat{y}) \leq[=][\geq] 0, \text { for } y<[=][>] \hat{y} .
$$

It follows that absolute prudence, $p(W)$, must be declining if $\operatorname{sgn} \frac{\partial Z(w)}{\partial w}$ is to be non-negative for any distribution of $y$. If $p(W)$ is increasing for some range of $W$, then there exists a binomial distribution of $y$ with

$$
\left\{\frac{E_{y}\left[u^{\prime \prime \prime}(W)\right]}{E_{y}\left[-u^{\prime \prime}(W)\right]}-p(W)\right\}(\Delta(y)-\Delta(\hat{y}))<0
$$

for both states, implying $\partial Z(w) / \partial w<0$. This establishes necessity of $p^{\prime}(W) \leq 0$. from

Sufficiency of declining prudence for $\operatorname{sgn} \frac{\partial Z(w)}{\partial w}$ to be non-negative follows

$$
\left\{\frac{E_{y}\left[u^{\prime \prime \prime}(W)\right]}{E_{y}\left[-u^{\prime \prime}(W)\right]}-p(W)\right\}(\Delta(y)-\Delta(\hat{y})) \geq 0, \forall y .
$$

Hence, declining absolute prudence is necessary and sufficient. 


\section{References}

Back, K., Dybvig, P. H.: On existence of optimal portfolios in complete markets. Washington University in St Louis, Working paper (1993)

Eeckhoudt, L., Gollier, C., Schlesinger, H.: The risk-averse (and prudent) newsboy. Management Science 41, 786-794 (1995)

Eeckhoudt, L., Gollier, C., Schlesinger, H.: Changes in background risk and risk taking behaviour. Econometrica 64, 683-689 (1996)

Eeckhoudt, L., Kimball, M. S.: Background risk, prudence, and the demand for insurance. In: Dionne, G. (ed.) Contributions to insurance economics, pp. 239-254. Boston: Kluwer 1992

Franke, G., Stapleton, R. C., Subrahmanyam, M. G.: Who buys and who sells options: the role of options in an economy with background risk. Journal of Economic Theory 82, 89-109 (1998)

Gollier, C.: The economics of risk and time. Cambridge, MA: MIT Press 2001

Gollier, C., Pratt, J. W.: Weak proper risk aversion and the tempering effect of background risk. Econometrica 64, 1109-1123 (1996)

Heaton, J., Lucas, D.: Portfolio choice in the presence of background risk. The Economic Journal 110, $1-26(2000)$

Hirshleifer, J., Riley, J.: The analytics of uncertainty and information. Cambridge: Cambridge University Press 1992

Kihlstrom R. E., Romer D., Williams S.: Risk aversion with random initial wealth. Econometrica 49, 911-920 (1981)

Kimball, M. S.: Precautionary saving in the small and in the large. Econometrica 58, 53-73 (1990)

Kimball, M. S.: Standard risk aversion. Econometrica 61, 589-611 (1993)

Meyer, D. J., Meyer, J.; Changes in background risk and the demand for insurance. The Geneva Papers on Risk and Insurance Theory 23, 29-40 (1998)

Meyer, J., Ormiston, M. B.: Deterministic transformations of random variables and the comparative statics of risk. Journal of Risk and Uncertainty 2, 179-188 (1989)

Nachman, D. C.: Preservation of 'more risk averse' under expectations. Journal of Economic Theory 28, 361-368 (1982)

Pratt, J. W.: Risk aversion in the small and in the large. Econometrica 32, 122-136 (1964)

Pratt, J. W., Zeckhauser, R. J.: Proper risk aversion. Econometrica 55, 143-154 (1987)

Viceira, L. M.: Optimal portfolio choice for long-horizon investors with nontradable labor income. Journal of Finance 56, 433-470 (2001)

Weil, P.: Equilibrium asset prices with undiversifiable labor income risk. Journal of Economic Dynamics and Control 16, 769-790 (1992) 